adverse effects can be avoided or used for the shortest possible duration.

Conflict of Interest None

\section{A COMPARISON OF PERCUTANEOUS AND SURGICAL REVASCULARISATION STRATEGIES FOLLOWING AN EQUIPOISE DECISION IN A HEART TEAM MEETING}

Jonathan Mailey, Mahmoud Ahmed, Michael Shahmohammadi, Conleth Murphy. Belfast Health and Social Care Trust, Belfast, UK

\subsection{6/heartjnl-2021-BCS.62}

Introduction Current guidelines advocate a Heart Team (HT) approach to decision making in complex coronary artery disease (CAD). Available evidence suggests that PCI is non-inferior to surgery in patients with three vessel or left main stem disease in the setting of a low-intermediate anatomical syntax score. The majority of HT discussions result in a recommendation of percutaneous coronary intervention (PCI) or coronary artery bypass surgery (CABG), however a decision is deemed to be equipoised when one revascularisation strategy is not felt to carry significant prognostic benefit. In this study we sought to evaluate clinical outcomes following an equipoise decision in a Heart Team meeting.

Methods This was a single centre retrospective study. All patients requiring intervention for structural heart disease in addition to CAD were excluded. Between January 2015 and December 2018 a total of 134 patients were deemed to be equipoise following discussion at a Heart Team meeting $(7.1 \%$ of all discussions). Demographics, procedural details and clinical outcomes were obtained from our national electronic health care record. The primary outcome was the 3-year rate of major adverse cardiovascular and cerebrovascular events (MACCE). This was a composite of cardiovascular death, nonprocedural myocardial infarction (MI), stroke and unplanned revascularisation.

Results A total of 108 patients underwent PCI and 26 CABG. Mean follow-up was $1177 \pm 384$ days in the PCI cohort and $1201 \pm 349$ days in the CABG cohort $(p=0.77)$. Baseline demographics did not differ significantly with the exception of chronic kidney disease and CAD involving 3 vessels (tables 1 $\& 2$ ). In the PCI cohort advanced calcium modification (intravascular lithotripsy or rotablation) was performed in $11.1 \%$ of procedures and intravascular imaging in 58.3\%. The mean number of stents used was $3.3 \pm 1.5$ and total stent length was

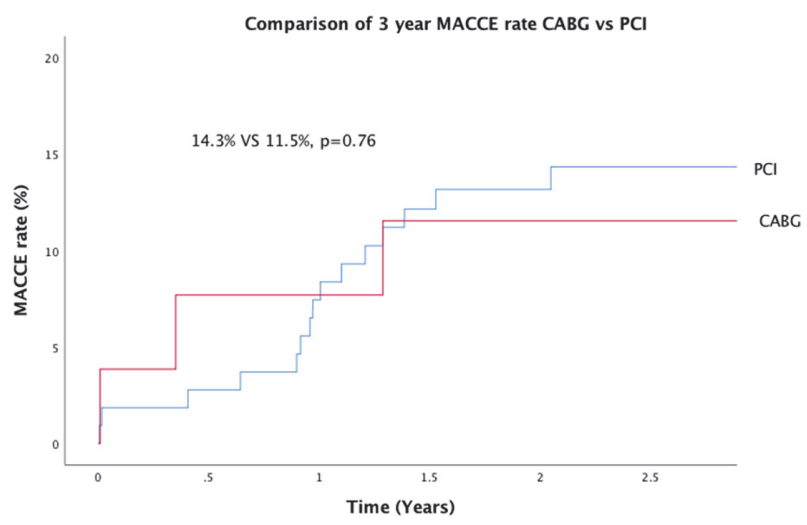

Abstract 62 Figure 1 Kaplan-Meier curve demonstrating a comparison of 3-Year MACCE rate between revascularisation modalities
Abstract 62 Table 1 Baseline demographics

\begin{tabular}{|c|c|c|c|}
\hline Variable & $\mathrm{PCI}(n=108)$ & CABG $(n=26)$ & $p$ value \\
\hline Age- mean+/-SD & $66.1+l-9.3$ & $63.9+1-7.0$ & 0.26 \\
\hline Male Gender- n (\%) & $88(81.5)$ & $22(84.6)$ & 1.0 \\
\hline \multicolumn{4}{|l|}{ Risk Factors- n (\%) } \\
\hline - Hypercholesterolaemia & $66(61.1)$ & $15(57.7)$ & 0.75 \\
\hline - Hypertension & $54(50.0)$ & $14(53.8)$ & 0.73 \\
\hline - Diabetes Mellitus & 19 (17.6) & $2(7.7)$ & 0.36 \\
\hline \multicolumn{4}{|l|}{ Co-morbidities- $n$ (\%) } \\
\hline - Stroke & $4(3.7)$ & $0(0.0)$ & 1.0 \\
\hline - PVD & $9(8.3)$ & $0(0.0)$ & 0.21 \\
\hline - COPD & $13(12.0)$ & $3(11.5)$ & 1.0 \\
\hline - Previous MI & $16(14.8)$ & $1(3.8)$ & 0.19 \\
\hline - Previous PCI & $20(18.5)$ & $1(3.8)$ & 0.08 \\
\hline - Previous cardiac surgery & $0(0.0)$ & $0(0.0)$ & 1.0 \\
\hline - CKD & 19 (17.6) & $0(0.0)$ & $\underline{0.02}$ \\
\hline
\end{tabular}

\begin{tabular}{|c|c|c|c|}
\hline Variable & $\mathrm{PCl}(n=108)$ & CABG $(n=26)$ & $p$ value \\
\hline LV function- $\mathrm{n}(\%)$ & & & 0.40 \\
\hline - Normal & $91(84.3)$ & $21(80.8)$ & \\
\hline - Mild & $12(11.1)$ & $2(14.3)$ & \\
\hline - Moderate & $4(3.7)$ & $3(11.5)$ & \\
\hline - Severe & $1(0.9)$ & $0(0.0)$ & \\
\hline Presentation- n (\%) & & & 0.65 \\
\hline - ACS & $51(47.2)$ & $12(46.2)$ & \\
\hline - Stable Angina & $57(52.8)$ & $14(53.8)$ & \\
\hline \multicolumn{4}{|c|}{ Syntax Score- mean+/-SD } \\
\hline - SS1 & $22.6+/-6.7$ & $24.4+/-7.1$ & 0.35 \\
\hline - SS2 PCl & $29.0+1-8.2$ & $27.5+l-6.4$ & 0.61 \\
\hline - SS2 CABG & $29.5+/-10.3$ & $26.9+1-6.5$ & 0.23 \\
\hline Syntax Tertile- n (\%) & & & 0.45 \\
\hline-1 & $57(52.8)$ & $11(42.3)$ & \\
\hline-2 & $42(38.9)$ & $11(42.3)$ & \\
\hline-3 & $9(8.3)$ & $4(15.4)$ & \\
\hline LMS disease- n (\%) & $56(51.9)$ & $12(46.2)$ & 0.60 \\
\hline \multicolumn{4}{|l|}{ Disease pattern- $n(\%)$} \\
\hline - 1 vessel & $8(7.4)$ & $0(0.0)$ & 0.35 \\
\hline - 2 vessel & $49(45.4)$ & $8(30.8)$ & 0.18 \\
\hline - 3 vessel & $51(47.2)$ & $18(69.2)$ & $\underline{0.04}$ \\
\hline
\end{tabular}

$97.0 \pm 47.5 \mathrm{~mm}$. The 3-year MACCE rate was $14.3 \%$ in the PCI cohort and $11.5 \%$ in the CABG cohort $(\mathrm{p}=0.76)$ (figure 1). 3-year cardiovascular death (PCI $2.8 \%$ vs CABG $0.0 \%$, $\mathrm{p}=0.39$ ) and unplanned revascularisation (PCI $11.5 \%$ vs CABG 7.9\%, $\mathrm{p}=0.62$ ) rates were numerically higher with PCI, whereas non-procedural MI (PCI 6.6\% vs CABG 7.7\%, $\mathrm{p}=0.85)$ and stroke (PCI $0.9 \%$ vs CABG 3.8\%, $\mathrm{P}=0.27$ ) rates were numerically higher with CABG. None of these differences met statistical significance. The incidence of stent thrombosis at 1 -year was $1.9 \%$ in the PCI cohort. PCI resulted in a reduction in total length of stay $(10.9 \pm 4.9$ days in the inpatient PCI vs $34.3 \pm 15.5$ days in the inpatient CABG cohorts, $\mathrm{p}<0.001)$.

Conclusions This study demonstrated no difference in MACCE between revascularisation modalities in patients with complex 
CAD deemed to be clinically equipoised following HT discussion. These results suggest that PCI is non-inferior to CABG in this population and provide validation to the decision making process of the HT in real life.

Conflict of Interest nil

\section{AN UNUSUAL CAUSE OF DYSPNOEA DIAGNOSED} DURING RIGHT HEART CATHETERISATION

James O'Brien, Rahul Chattopadhyay, Peter J Pugh. Cambridge University Hospital, Cambridge, UK

\subsection{6/heartjnl-2021-BCS.63}

Background It is common for patients with exertional dyspnoea to be referred to Cardiology for investigation. This case highlights a rare but important cause of cyanosis, diagnosed after extensive investigations.

Case Presentation A 73-year-old was referred to the Respiratory Medicine team with limiting exertional dyspnoea. Initial standard investigations proved unremarkable, with normal spirometry, CT pulmonary angiogram, V/Q scan, 6-minute walk test and normal $\mathrm{PaO} 2$ on arterial blood gas sampling. The patient was then referred to Cardiology for consideration of a cardiac cause of her symptoms. Her past medical history included rheumatoid arthritis, retinal vein thrombosis and idiopathic right arm thrombosis. After her initial review by Cardiology, a decision was made to perform both a coronary angiogram and right heart catheterisation. The coronary arteries showed no significant flow limiting lesion and the pulmonary pressures were not significantly elevated with no evidence of a shunt During the procedure, it was noted that the patient's blood was dark brown in colour (figure 1), with low systemic (66\%) and pulmonary artery $(48.6 \%)$ oxygen saturation as measured on the cath lab oximeter, with normal respiratory rate (12/minute). Arterial blood was mixed in a syringe with $100 \%$ oxygen, which produced no change in the colour. A full blood gas analysis was therefore undertaken, which identified a disparity between the measured $\mathrm{PaO} 2(12.0 \mathrm{kPa})$ and oxygen saturations. A haemoglobinopathy was therefore suspected, which was felt most likely to be a case of methaemoglobinaemia. However, it proved not possible to measure this on the multiple point of care ABG machines, nor at the main biochemistry laboratory. The patient was subsequently investigated extensively by Haematology, ultimately identifying diagnosis of sulfhaemoglobinaemia. The source for the sulphated haemoglobin was due to an Epsom salt (MgSO4) containing tonic the patient was using for constipation. The patient was treated with exchange transfusions under the care of the haematology team alongside stopping the Epsom salt tonic.

Discussion Sulfhaemoglobinaemia (SulfHb) is a rare haemoglobinopathy which arises due to the sulphation of haemoglobin, this results in a significantly reduced oxygen carrying capacity due to reduced affinity for oxygen (Lu et al., 1998); it is often reported that the source of exogenous sulphur is from medications, bowel pathology or occupational exposures. This case highlights the importance of right heart catheterisation as an investigation to help differentiate the causes of exertional dyspnoea and the importance of considering full blood gas analysis when the numbers do not add up.

Conclusion Sulfhaemoglobinaemia is a rare cause of exertional dyspnoea and should be considered in patients with a clear

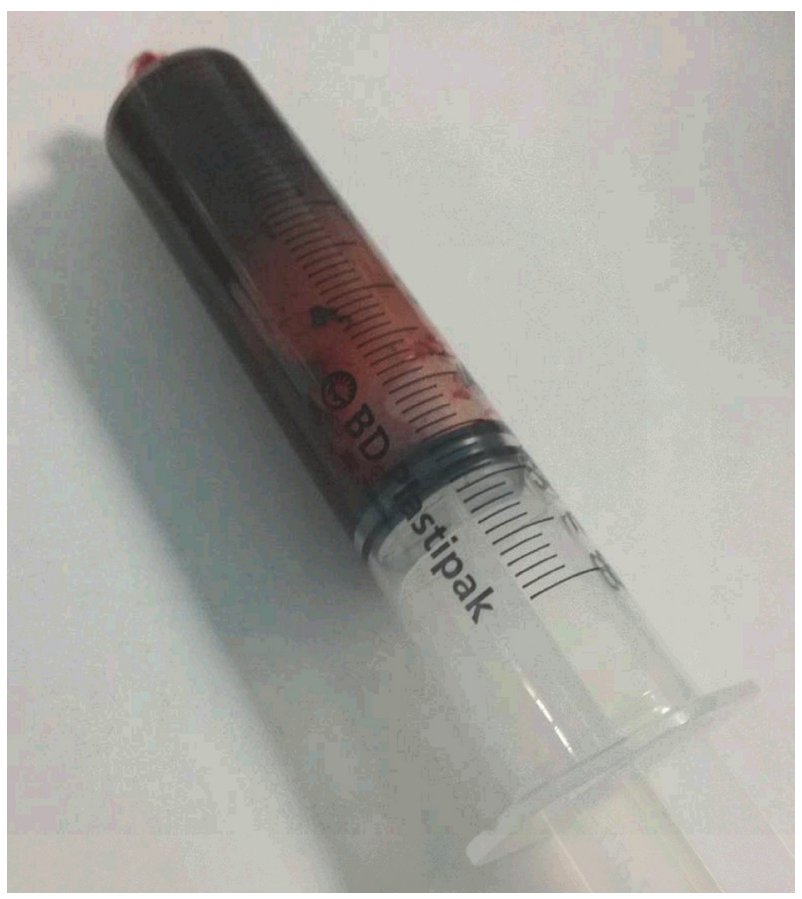

Abstract 63 Figure 1

disparity between saturations measured by pulse oximetry and atrial blood sampling.

Conflict of Interest None

\section{USE OF SIROLIMUS-COATED BALLOON IN DE NOVO LESIONS; MID-TERM FOLLOW-UP FROM A SINGLE- CENTRE REGISTRY}

Bhagya Harindi Loku Waduge, Harkaran Kalkat, Ameenathul Mazaya Fawzy, Abdullah Saif, Sampath Athukorala, Gurbir Bhatia, Nitin Kumar, Bethan Freestone, Jerome Ment Kaeng Lee, Michael Pitt, George Pulikal, Sandeep Basavarajaiah Sandeep Basavarajaiah. Birmingham Heartlands Hospital, Birmingham, UK

\subsection{6/heartjnl-2021-BCS.64}

Aims Use of drug coated balloon (DCB) in coronary intervention is escalating and the guidelines recommend its use in restenotic lesions. However, some of the recent data suggest, it can also be considered in subset of denovo lesions especially; small vessels, ostium of an important side-branch and in patients unable to tolerate dual antiplatelet therapy. Sirolimus is the drug of choice in currently available drug eluting stents. Most DCBs used elute Paclitaxcel, but there is no data on Sirolimus coated DCBs. In this study, we report outcomes from the use of a Sirolimus coated balloon (SCB) in de novo coronary lesions.

Methods We included all patients treated with an SCB between April and December 2018. Outcomes measured include cardiac death, target vessel myocardial infarction, target lesion revascularisation, target vessel revascularisation and MACE (combination of cardiac death, target vessel MI and TLR).

Results During the study period, 351 patients (with 414 lesions) with de novo lesions were treated with an SCB. The mean age of patients was $65.6 \pm 11.5$ years, 275 (78\%) were male and $39 \%(n=212)$ had diabetes. Most lesions treated were in the LAD/diagonal system $(n=173,42 \%)$. Predilatation 